Network Working Group

Request for Comments: 5377

Category: Informational
J. Halpern, Ed.

Self

November 2008

Status of This Memo

This memo provides information for the Internet community. It does not specify an Internet standard of any kind. Distribution of this memo is unlimited.

Copyright Notice

Copyright (c) 2008 IETF Trust and the persons identified as the document authors. All rights reserved.

This document is subject to BCP 78 and the IETF Trust's Legal Provisions Relating to IETF Documents

(http://trustee.ietf.org/license-info) in effect on the date of publication of this document. Please review these documents carefully, as they describe your rights and restrictions with respect to this document.

Abstract

Contributors grant intellectual property rights to the IETF. The IETF Trust holds and manages those rights on behalf of the IETF. The Trustees of the IETF Trust are responsible for that management. This management includes granting the licenses to copy, implement, and otherwise use IETF Contributions, among them Internet-Drafts and RFCs. The Trustees of the IETF Trust accepts direction from the IETF regarding the rights to be granted. This document describes the desires of the IETF regarding outbound rights to be granted in IETF Contributions. 
Table of Contents

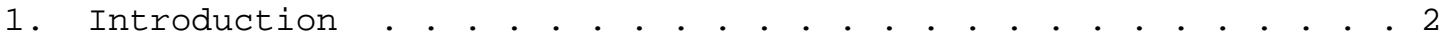

2. Purpose in Granting Rights . . . . . . . . . . . . . . . . . . 2

3. Powers and Authority . . . . . . . . . . . . . . . . . . . . . 3

4. Recommended Grants of Right to Copy . . . . . . . . . . . . . . 4 4.1. Rights Granted for Reproduction of RFCs . . . . . . . . . 5

4.2. Rights Granted for Quoting from IETF Contributions . . . 5

4.3. Rights Granted for Implementing Based on IETF

Contributions . . . . . . . . . . . . . . . . . 5

4.4. Rights Granted for Use of Text from IETF Contributions . • 6

4.5. Additional Licenses for IETF Contributions . . . . . . . 6

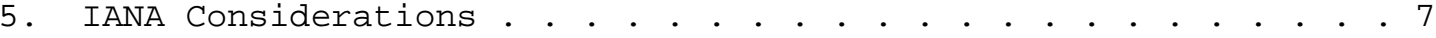

6. Security Considerations . . . . . . . . . . . . . . . . . . 7

7. References . . . . . . . . . . . . . . . . . . . . . 7

7.1. Normative References . . . . . . . . . . . . . . . . 7

7.2. Informative References . . . . . . . . . . . . . . . . . . 7

1. Introduction

Under the current operational and administrative structures, IETF intellectual property rights are vested in the IETF Trust administered by a board of trustees made up of the members of the IAOC [RFC4371]. This includes the right to make use of IETF

Contributions, as granted by Contributors under the rules laid out in [RFC5378]. The Trustees of the IETF Trust are therefore responsible for defining the rights to copy granted by the IETF to people who wish to make use of the material in these documents.

For consistency and clarity, this document uses the same terminology laid out in [RFC5378] and uses the same meanings as defined in that document.

The IETF Trust, by way of its Trustees, has indicated, as is consistent with the IETF structure, that it will respect the wishes of the IETF in regard to what these granted rights ought to be. It is therefore the IETF's responsibility to articulate those wishes. This document represents the wishes of the IETF regarding the rights granted to all users in regard to IETF Contributions, until it is superseded.

2. Purpose in Granting Rights

In providing a description of the wishes of the IETF with regard to rights granted in RFCs, it is helpful to keep in mind the purpose of granting such rights. 
The mission of the IETF is to produce documents that make the Internet work better (see [RFC3935] for more details). These documents, when completed, are published as RFCs.

An important subclass of RFCs is standards describing protocols; for these, the primary value to the Internet is the ability of implementors to build solutions (products, software, etc.) that interoperate using these standards. Hence, the IETF has a strong interest in seeing accurate, interoperable implementations of the material the IETF publishes. The IETF Trust grants rights to copy to people to make use of the text in the RFCs in order to encourage accurate and interoperable implementations.

As early implementations from Internet-Drafts make use of descriptions in those Internet-Drafts, similar desires apply to Internet-Drafts.

Similar considerations also apply to non-standard, non-protocol documents such as BCP (Best Current Practice) and Informational documents; in this document, we recommend a common approach to the issue of right-to-use licenses for all IETF documents.

Previous documents regarding rights in IETF documents have included in the RFC text specific text to be used to achieve the stated goals. This has proved problematic. When problems are found with such text, even when the problem is not a change in intent, it is necessary to revise the RFC to fix the problem. At best, this delays fixing legal issues that need prompt attention. As such, this document describes the IETF desires to the Trustees of the IETF Trust, but does not provide the specific legal wording to address the goals. The selection, and updating as necessary, of legal wording is left to the Trustees of the IETF Trust. Appeals of the actions of the Trustees of the IETF Trust are governed by other documents. As the Trustees are the members of the IAOC, the appeals procedure documented in BCP 101 (currently [RFC4371]) is applicable.

\section{Powers and Authority}

As described in the introduction, and formally specified in [RFC5378], the legal authority for determining and granting users rights to copy material in RFCs and other IETF Contributions rests with the Trustees for the IETF Trust, which is made up of the members of the IAOC, as described in [RFC4071] and [RFC4371]. This document provides guidance to that body, based on the rough consensus of the IETF. The Trustees of the IETF Trust have the authority and responsibility to determine the exact text insertions (or other mechanisms), if any, needed in Internet-Drafts, RFCs, and all IETF 
Contributions to meet these goals. The IETF Trust License Policy is available from http://trustee.ietf.org/license-info.

The rough consensus described in this document reflects the agreement of the IETF as of the IETF Last Call, and the Trustees of the IETF Trust are to begin drafting license text and other materials to act on these instructions upon IESG approval of this document for RFC publication. Changes to the IETF documentation, and document policies themselves, take effect as determined by the Trustees of the IETF Trust.

This document does not specify what rights the IETF Trust receives from others in IETF Contributions. That is left to another document ([RFC5378]). While care has been taken by the working group in developing this document, and care will be taken by the Trustees of the IETF Trust, to see that sufficient rights are granted to the IETF Trust in IETF Contributions, it is also the case that the Trust can not grant rights it has not or does not receive, and it is expected that policies will be in line with that fact. Similarly, the rights granted for pre-existing documents can not be expanded unless the holders of rights in those Contributions choose to grant expanded rights. Nonetheless, to the degree it can, and without embarking on a massive effort, it is desirable if similar rights to those described below can be granted in older RFCs.

\section{Recommended Grants of Right to Copy}

The IETF grants rights to copy and modify parts of IETF Contributions in order to meet the objectives described earlier. As such, different circumstances and different parts of documents may need different grants. This section contains subsections for each such different grant that is currently envisioned. Each section is intended to describe a particular usage, to describe how that usage is recognizable, and to provide guidance to the Trustees of the IETF Trust as to what rights the IETF would like to see granted in that circumstance and what limitations should be put on such granting.

These recommendations for outgoing rights are structured around the assumptions documented in [RFC5378]. Thus, this document is about granting rights derived from those granted to the IETF Trust. The recommendations below are how those granted rights should in turn be passed on to others using IETF documents in ways and for purposes that fit with the goals of the IETF. This discussion is also separate from discussion of the rights the IETF itself requires in documents to do its job, as those are not "outbound" rights. It is expected that the rights granted to the IETF will be a superset of those copying rights we wish to grant to others. 


\subsection{Rights Granted for Reproduction of RFCs}

It has long been IETF policy to encourage copying of RFCs in full. This permits wide dissemination of the material, without risking loss of context or meaning. The IETF wishes to continue to permit anyone to make full copies and translations of RFCs.

\subsection{Rights Granted for Quoting from IETF Contributions}

There is rough consensus that it is useful to permit quoting without modification of excerpts from IETF Contributions. Such excerpts may be of any length and in any context. Translation of quotations is also to be permitted. All such quotations should be attributed properly to the IETF and the IETF Contribution from which they are taken.

\subsection{Rights Granted for Implementing Based on IETF Contributions}

IETF Contributions often include components intended to be directly processed by a computer. Examples of these include ABNF definitions, XML Schemas, XML DTDs, XML RelaxNG definitions, tables of values, MIBS, ASN.1, and classical programming code. These are included in IETF Contributions for clarity and precision in specification. It is clearly beneficial, when such items are included in IETF Contributions, to permit the inclusion of such code components in products that implement the Contribution. It has been pointed out that in several important contexts, use of such code requires the ability to modify the code. One common example of this is simply the need to adapt code for use in specific contexts (languages, compilers, tool systems, etc.) Such use frequently requires some changes to the text of the code from the IETF Contribution. Another example is that code included in open source products is frequently licensed to permit any and all of the code to be modified. Since we want this code included in such products, it follows that we need to permit such modification. While there has been discussion of restricting in some way the rights to make such modifications, the rough consensus of the IETF is that such restrictions are likely a bad idea, and are certainly very complex to define.

As such, the rough consensus is that the IETF Trust is to grant rights such that code components of IETF Contributions can be extracted, modified, and used by anyone in any way desired. To enable the broadest possible extraction, modification, and usage, the IETF Trust should avoid adding software license obligations beyond those already present in a Contribution. The granted rights to extract, modify, and use code should allow creation of derived works outside the IETF that may carry additional license obligations. As the IETF Trust can not grant rights it does not receive, the rights 
to extract, modify, and use code described in this paragraph can not be granted in IETF Contributions that are explicitly marked as not permitting derivative works.

While it is up to the Trustees of the IETF Trust to determine the best way of meeting this objective, two mechanisms are suggested here that are believed to be helpful in documenting the intended grant to readers and users of IETF Contributions.

Firstly, the Trustees of the IETF Trust should maintain, in a suitable, easily accessible fashion, a list of common RFC components that will be considered to be code. To start, this list should include at least the items listed above. The Trustees of the IETF Trust will add to this list as they deem suitable or as they are directed by the IETF.

Additionally, the Trustees of the IETF Trust should define a textual representation to be included in an IETF Contribution to indicate that a portion of the document is considered by the authors (and later, the working group, and upon approval, the IETF) to be code and thus subject to the permissions granted to use code.

4.4. Rights Granted for Use of Text from IETF Contributions

There is no consensus at this time to permit the use of text from RFCs in contexts where the right to modify the text is required. The authors of IETF Contributions may be able and willing to grant such rights independently of the rights they have granted to the IETF by making the Contribution.

\subsection{Additional Licenses for IETF Contributions}

There have been contexts where the material in an IETF Contribution is also available under other license terms. The IETF wishes to be able to include content that is available under such licenses. It is desirable to indicate in the IETF Contribution that other licenses are available. It would be inappropriate and confusing if such additional licenses restricted the rights the IETF intends to grant in the content of RFCS.

However, the IETF does not wish to have IETF Contributions contain additional licenses, as that introduces a number of additional difficulties. Specifically, additional text in the document, and any additional license referred to by permitted additional text, must not in any way restrict the rights the IETF intends to grant to others for using the contents of IETF Contributions. 
Authors of Contributions retain all rights in their contributions. As such, an author may directly grant any rights they wish separately from what the IETF grants. However, a reader wishing to determine or make use of such grants will need to either consult external sources of information, possibly including open source code and documents, or contact the author directly.

5. IANA Considerations

No values are assigned in this document, no registries are created, and there is no action assigned to the IANA by this document. One list (of kinds of code sections) is anticipated, to be created and maintained by the Trustees of the IETF Trust. It is up to the Trustees of the IETF Trust whether they create such a list and whether they choose to involve the IANA in maintaining that list.

6. Security Considerations

This document introduces no new security considerations. It is a process document about the IETF'S IPR rights being granted to other people. While there may be attacks against the integrity or effectiveness of the IETF processes, this document does not address such issues.

7. References

7.1. Normative References

[RFC5378] Bradner, S., Ed. and J. Contreras, Ed., "Rights Contributors Provide to the IETF Trust", BCP 78, RFC 5378, November 2008 .

\subsection{Informative References}

[RFC3935] Alvestrand, H., "A Mission statement for the IETF", $\mathrm{BCP}$ 95, RFC 3935, October 2004.

[RFC4071] Austein, R. and B. Wijnen, "Structure of the IETF Administrative Support Activity (IASA)", BCP 101, RFC 4071, April 2005.

[RFC4371] Carpenter, B. and L. Lynch, "BCP 101 Update for IPR Trust", BCP 101, RFC 4371, January 2006. 


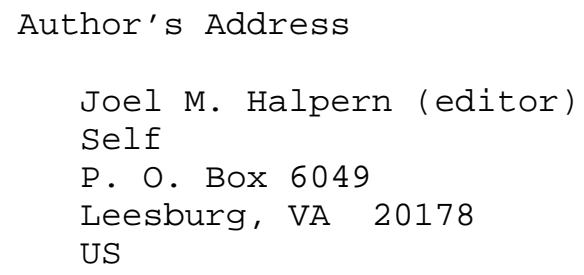

EMail: jmh@joelhalpern.com 